\title{
Scan Time Frame
}

National Cancer Institute

\section{Source}

National Cancer Institute. Scan Time Frame. NCI Thesaurus. Code C94975.

A time interval within the total duration of a scan at one bed position during which detector data is acquired for an image. In the usual single scan there is only one frame at each bed position, with these occurring some chosen times after injection. In dynamic scans however there are a number of sequential frames at a fixed bed position. 